\title{
CULTIVO DE TAMBAQUI EM GAIOLAS DE BAIXO VOLUME: EFEITO DA DENSIDADE DE ESTOCAGEM NA PRODUÇÃO DE BIOMASSA
}

\author{
Luis ANTONio Kioshi AOKi INOUE ${ }^{1}$, Alcimara CHAMES BEZERRA ${ }^{2}$, WiLliam SANDRO \\ MIRANDA $^{2}$, AleKSANDER WestPHAl MUNIZ ${ }^{3}$, CHEILA DE LIMA BOIJINK ${ }^{3}$ \\ ${ }^{1}$ Pesquisador Doutor da Embrapa Agropecuária Oeste, Dourados, MS, Brasil - luis.inoue@embrapa.br \\ ${ }^{2}$ Bolsistas Programa de Apoio à Iniciação Científica do Amazonas (PAIC/Fapeam), Embrapa Amazônia Ocidental, \\ Manaus, AM, Brasil \\ ${ }^{3}$ Pesquisadores Doutores Embrapa Amazônia Ocidental, Manaus, AM, Brasil.
}

A criação de peixes em gaiolas flutuantes na Amazônia Ocidental é um assunto de interesse inclusive governamental, pois traz à discussão a possibilidade do uso sustentável de grandes corpos de água da bacia amazônica, podendo, assim, beneficiar populações ribeirinhas com geração de trabalho e renda. O tambaqui é umas das espécies mais indicadas para esse fim por ser um peixe onívoro, bem apreciado no mercado consumidor local, além da demanda crescente, devido à redução dos estoques naturais pela pesca extrativista e desmatamento da mata ciliar nos arredores das grandes cidades nortistas como Manaus. Entretanto, mais informações básicas sobre a criação da espécie em gaiolas de baixo volume ainda são necessárias para o aperfeiçoamento e avanço da atividade. $\mathrm{O}$ presente trabalho estudou os efeitos de quatro densidades de estocagem $\left(10,20,30\right.$ e 40 peixes $\left./ \mathrm{m}^{3}\right)$ no crescimento e na incidência de parasitas, monogêneas, nas brânquias do tambaqui cultivado em gaiolas de baixo volume $\left(1 \mathrm{~m}^{3}\right)$. O estudo pode contribuir com mais informações elementares para a exploração racional dos recursos naturais amazônicos, o tambaqui e os grandes corpos de água. Os resultados mostram que é possível aumentar a produção de biomassa de tambaqui em gaiolas de baixo volume de $1 \mathrm{~m}^{3}$ aumentando-se a densidade de estocagem até 40 peixes $/ \mathrm{m}^{3}$, sem prejuízos no crescimento e aumento de carga parasitária nas brânquias dos peixes.

PALAVRAS-CHAVE: Colossoma macropomum; crescimento; parasitas ; piscicultura.

\section{TAMBAQUI FARMING IN SMALL VOLUME CAGES: EFFECTS OF STOCKING DENSITY ON THE BIOMASS PRODUCTION}

\section{ABSTRACT}

Fish farming in small floating cages is the subject of local discussions due to the possible sustainable use of the Amazon basin, alleviating poverty of people who live near lakes and rivers. "Tambaqui" is one of the main species indicated to this end because this is an omnivorous species, well accepted in the local market, whose demand grows fast due to the over-fishing around big western Amazon cities such as Manaus. However, more information about "tambaqui" farming in small volume cages is necessary to increase and advance production in this system. Therefore, we studied the effects of four stocking densities $\left(10,20,30\right.$ and $\left.40 \mathrm{fish} / \mathrm{m}^{3}\right)$ in "tambaqui" farming in small cages $\left(1 \mathrm{~m}^{3}\right)$. We evaluated fish growth and number of gills parasites. The results showed clear fish biomass rise by the increase of "tambaqui" stocking density. No effect of stocking density was observed on fish individual growth, survival and number of gills parasites.

KEYWORDS: Colossoma macropomum; fish-farming; growth; parasites. 


\section{INTRODUÇÃO}

O cultivo de peixes em gaiolas flutuantes na Amazônia Ocidental tem sido assunto de grande interesse entre técnicos da piscicultura e articuladores de políticas públicas, devido à possibilidade de uso sustentável de grandes corpos de água da bacia amazônica, promovendo a inclusão social de populações ribeirinhas com geração de trabalho e renda ${ }^{1}$.

Para tal atividade, uma das espécies com características positivas é o tambaqui, pois é um dos peixes mais comercializados nos mercados dos centros urbanos da região norte, a preços desejáveis com demanda ainda crescente, devido ao aumento das populações consumidoras e diminuição dos estoques naturais da espécie nos arredores de grandes cidades amazônicas como Manaus ${ }^{1}$. Entretanto, o cultivo do tambaqui em gaiolas é uma atividade relativamente recente na região e mais estudos são necessários sobre, por exemplo, as máximas produções possíveis nos diversos ambientes aquáticos e tipos de instalações, densidades econômicas e gerenciamento ambientalmente amigável ${ }^{1}$. Alguns autores já testaram o cultivo do tambaqui em gaiolas de $6 \mathrm{~m}^{3}$ com resultados promissores em um lago formado pelas águas da cheia do rio Amazonas no município de Iranduba, $\mathrm{AM}^{1,2}$.

De maneira a contribuir com as informações existentes, o presente trabalho avaliou os efeitos da densidade de estocagem no crescimento e carga parasitária nas brânquias do tambaqui, cultivado em gaiolas flutuantes de baixo volume $\left(1 \mathrm{~m}^{3}\right)$. Adotou-se o termo densidade de estocagem como a quantidade de peixes colocada em uma determinada gaiola no início de um período de alimentação artificial e completa, com avaliação regular do crescimento dos animais, em condições similares de um cultivo comercial ou de subsistência, que são usualmente estimulados pelas agências locais de fomento.

\section{MATERIAL E MÉTODOS}

Trezentos tambaquis jovens com peso médio de $150,2 \pm 32,7 \mathrm{~g}$ e comprimento médio de $20,5 \pm$ $5,2 \mathrm{~cm}$ foram estocados em 12 gaiolas de $1 \mathrm{~m}^{3}$, em densidades de $10,20,30$ e 40 peixes $/ \mathrm{m}^{3}$, montadas em um açude de 8 ha $\left(2^{\circ} 511^{\prime} 34,66^{\prime}\right.$ 'S; $59^{\circ} 56^{\prime} 43,41^{\prime}$ 'O), de uma propriedade particular nas imediações de Manaus, AM. Dessa forma, foram estabelecidos quatro tratamentos com três repetições cada, em delineamento inteiramente casualizado. Variáveis da qualidade da água foram monitoradas duas vezes por semana sempre no início do período da manhã: temperatura $\left({ }^{\circ} \mathrm{C}\right)$ e oxigênio $(\mathrm{mg} / \mathrm{L})$ em medidor de concentração de oxigênio marca YSI modelo 55, pH em medidor YSI de campo; amostra de água era tomada próximo à superfície para determinação, em laboratório, da alcalinidade $\left(\mathrm{CaCO}_{3} / \mathrm{L} \mathrm{mg} / \mathrm{L}\right)$ e dureza total $\left(\mathrm{CaCO}_{3} / \mathrm{L} \mathrm{mg} / \mathrm{L}\right)$ por titulação com ácido sulfúrico e EDTA diluídos, respectivamente, e determinação da amônia total $(\mathrm{mg} / \mathrm{L})$ pelo método fenol com leitura em espectrofotômetro.

Os peixes foram alimentados duas vezes ao dia com ração extrusada comercial, contendo $32 \%$ de proteína bruta, durante 120 dias, entre os meses de agosto a novembro, até a saciedade aparente.

No início do trabalho e a cada mês todos os peixes de todas as gaiolas foram levemente anestesiados com eugenol ${ }^{3}$, pesados (g) e medidos (cm). A partir desses valores foram calculados para cada gaiola:

1. Biomassa inicial $\left(\mathrm{kg} / \mathrm{m}^{3}\right)=$ somatório do peso individual no início do experimento $(\mathrm{g}) / 1000$

2. Biomassa final $\left(\mathrm{kg} / \mathrm{m}^{3}\right)=$ somatório peso individual no final do experimento $(\mathrm{g}) / 1000$

3. Ganho de biomassa $\left(\mathrm{kg} / \mathrm{m}^{3}\right)=$ Biomassa final $\left(\mathrm{kg} / \mathrm{m}^{3}\right)$ - Biomassa inicial $\left(\mathrm{kg} / \mathrm{m}^{3}\right)$

4. Ganho diário de biomassa $\left(\mathrm{kg} / \mathrm{m}^{3}\right.$ dia $)=[$ Ganho biomassa $\left(\mathrm{kg} / \mathrm{m}^{3}\right) / 120$ dias $]^{*} 1000$

5. Ganho de peso $(\mathrm{g})=$ Peso médio final $(\mathrm{g})-$ Peso médio inicial $(\mathrm{g})$

6. Ganho de peso diário $(\mathrm{g} / \mathrm{dia})=$ Ganho peso (g)/120 dias

7. Taxa de crescimento específico $=[(\ln$ Peso médio final $-\ln$ Peso médio inicial)/120dias]*100

8 . Sobrevivência $=($ número de peixes no final do experimento/número de peixes inicial) $* 100$

9. Conversão alimentar aparente $=$ Quantidade de ração fornecida $(\mathrm{kg}) / \mathrm{Ganho}$ de biomassa $\left(\mathrm{kg} / \mathrm{m}^{-3}\right)$.

Dessa forma, os índices calculados acima permitiram avaliar os efeitos da densidade de estocagem no crescimento e incremento de biomassa do tambaqui em gaiolas de baixo volume.

Ao final de 120 dias, três animais de cada gaiola, totalizando nove peixes por tratamento, foram coletados, anestesiados e sacrificados por perfuração da fontanela craniana. O projeto foi aprovado pela comissão de ética no uso de animais - CEUA/UFGD (Protocolo $\mathrm{n}^{\circ}$. 003/2014). Os peixes foram examinados macroscopicamente para verificação de eventuais alterações na superfície do corpo. Logo em 
seguida, as brânquias foram removidas, cada arco destacado e o conjunto de arcos branquiais fixado em formol $(5 \%)$ em frascos individuais, para posterior contagem de monogenóides em cada arco branquial com auxílio de microscópio estereoscópico.

Os dados de crescimento, incremento de biomassa e carga parasitária do tambaqui em gaiolas de baixo volume foram submetidos à análise de variância e, quando $\mathrm{F}$ significativo, foi feito ajuste de regressão. $\mathrm{O}$ nível de significância adotado foi de $5 \%$.

\section{RESULTADOS E DISCUSSÃO}

As variáveis da qualidade da água permaneceram dentro das condições normais ${ }^{4}$ para a criação de peixes tropicais (temperatura $29,0^{\circ} \mathrm{C}, \mathrm{pH}$ 7,14 , oxigênio $6,6 \mathrm{mg} / \mathrm{L}$, dureza $8,3 \mathrm{mg} / \mathrm{L}$, alcalinidade $12,7 \mathrm{mg} / \mathrm{L}$, amônia total $0,03 \mathrm{mg} / \mathrm{L}$ ). Os efeitos da densidade de estocagem no crescimento do tambaqui em gaiolas flutuantes de baixo volume estão expressos na Tabela 1 e Figura 1 em média e desvio padrão da média. Não foram observadas diferenças significativas entre tratamentos para os valores de peso, comprimento, ganho de peso, ganho de peso diário, taxa de crescimento específico, sobrevivência, alimento fornecido e conversão alimentar aparente.

Tabela 1. Efeito da densidade de estocagem em índices zootécnicos e carga parasitária de brânquias de tambaqui cultivado em gaiolas de baixo volume $\left(1 \mathrm{~m}^{3}\right)$ na Amazônia Ocidental de agosto a novembro (120 dias)

\begin{tabular}{lcccc}
\hline & \multicolumn{4}{c}{ Densidade de estocagem (peixes $\left./ \mathrm{m}^{3}\right)$} \\
\cline { 2 - 5 } & 10 & 20 & 30 & 40 \\
\hline Biomassa final $\left(\mathrm{kg} / \mathrm{m}^{3}\right)$ & $5,2 \pm 0,8$ & $11,5 \pm 3,4$ & $13,9 \pm 3,3$ & $18,6 \pm 6,6$ \\
Ganho biomassa no período & $3,8 \pm 0,3$ & $7,9 \pm 3,2$ & $9,8 \pm 3,5$ & $13,0 \pm 3,9$ \\
$\left(\mathrm{~kg} / \mathrm{m}^{3}\right)$ & & & & \\
Ganho diário biomassa & $32 \pm 3$ & $65 \pm 27$ & $82 \pm 29$ & $108 \pm 3$ \\
$\left(\mathrm{~g} / \mathrm{m}^{3}\right.$ dia) & & & & \\
Ganho de peso (g) & $440 \pm 31$ & $430 \pm 27$ & $425 \pm 44$ & $336 \pm 66$ \\
Ganho de peso diário (g/dia) & $3,7 \pm 0,3$ & $3,6 \pm 1,3$ & $3,5 \pm 0,4$ & $2,8 \pm 0,5$ \\
Ração fornecida (kg) & $6,9 \pm 0,5$ & $24,4 \pm 0,3$ & $34,5 \pm 3,7$ & $33,1 \pm 2,8$ \\
Taxa de crescimento & $1,0 \pm 0,3$ & $0,7 \pm 0,3$ & $0,9 \pm 0,1$ & $0,7 \pm 0,1$ \\
específico (\%) & & & & \\
Sobrevivência (\%) & $90 \pm 10$ & $93 \pm 3$ & $82 \pm 17$ & $98 \pm 1,8$ \\
Conversão alimentar & $1,8 \pm 0,0$ & $3,1 \pm 0,3$ & $3,5 \pm 0,4$ & $2,1 \pm 0,6$ \\
Parasitos nas brânquias & $32 \pm 6$ & $46 \pm 5$ & $77 \pm 9$ & $60 \pm 16$ \\
\hline
\end{tabular}

Coeficiente de variação $=34 \%$

No entanto o ajuste de regressão mostra que a biomassa final foi mais alta no tratamento de 40 peixes $/ \mathrm{m}^{3}$, ainda com melhores índices de ganho de biomassa e ganho diário de biomassa (Figura 2). Assim, este estudo demonstrou que, aumentando-se a densidade de estocagem do tambaqui em gaiolas de baixo volume $\left(1 \mathrm{~m}^{3}\right)$, é possível alcançar maior biomassa final de peixes, sem alterações significativas dos parâmetros zootécnicos como peso, comprimento, ganho de peso, ganho de peso diário, taxa de crescimento específico, sobrevivência e conversão alimentar aparente.

Dessa forma, o aumento da densidade de estocagem no tipo de gaiola em estudo $\left(1 \mathrm{~m}^{3}\right)$ possibilitou maior produção de biomassa, sem limitações zootécnicas para a espécie, tal como observado por Gomes et al. ${ }^{1}$ em cultivo de tambaqui em gaiolas de $6 \mathrm{~m}^{3}$. Esses autores submeteram juvenis de tambaqui a densidades de estocagem de até 50 peixes $/ \mathrm{m}^{3}$ por 240 dias e não encontraram limitações de crescimento e conversão alimentar aparente do tambaqui em gaiolas maiores. Ainda de acordo com os autores, o tambaqui não atingiu a capacidade de suporte do sistema. No entanto, o que limitaria o cultivo de tambaqui em gaiolas de $6 \mathrm{~m}^{3}$ seriam os fatores como o preço da ração versus o preço de venda dos peixes, e máximo input permitido de nutrientes no sistema, que determinam os índices de biomassa crítica, biomassa ambientalmente sustentável e biomassa econômica ${ }^{4}$.

$\mathrm{O}$ aumento da densidade de estocagem a 
níveis limítrofes conhecidamente diminui o crescimento, devido ao aumento da competição entre os animais na alimentação e diminuição do consumo individual de alimento ${ }^{5}$, estresse social ${ }^{6}$ e maior contato entre os animais favorecendo a disseminação I de parasitos ${ }^{6}$. Todos os peixes analisados estavam parasitados nas brânquias com monogenóides com prevalência de $100 \%$. A densidade de estocagem de 30 peixes $/ \mathrm{m}^{3}$ apresentou os maiores números de parasitos nas brânquias, porém as diferenças não foram significativas (Tabela 1), ou seja, não foi observado maior carga de parasitos nas brânquias do tambaqui por efeito da maior densidade de estocagem.

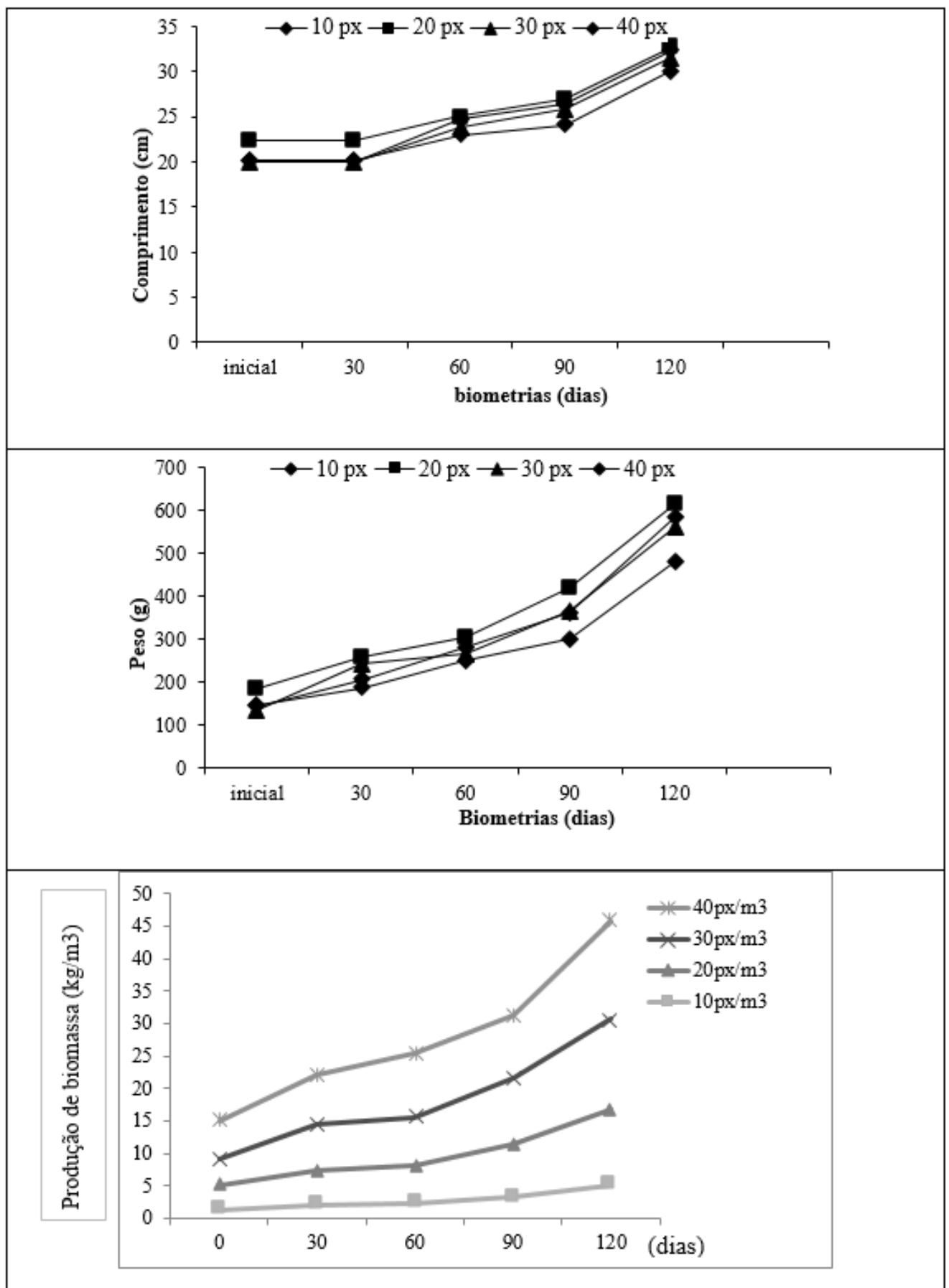

Figura 1. Crescimento do tambaqui estocado em gaiolas de baixo volume $\left(1 \mathrm{~m}^{3}\right)$ com diferentes densidades de estocagem na Amazônia Ocidental durante cultivo de quatro meses. 


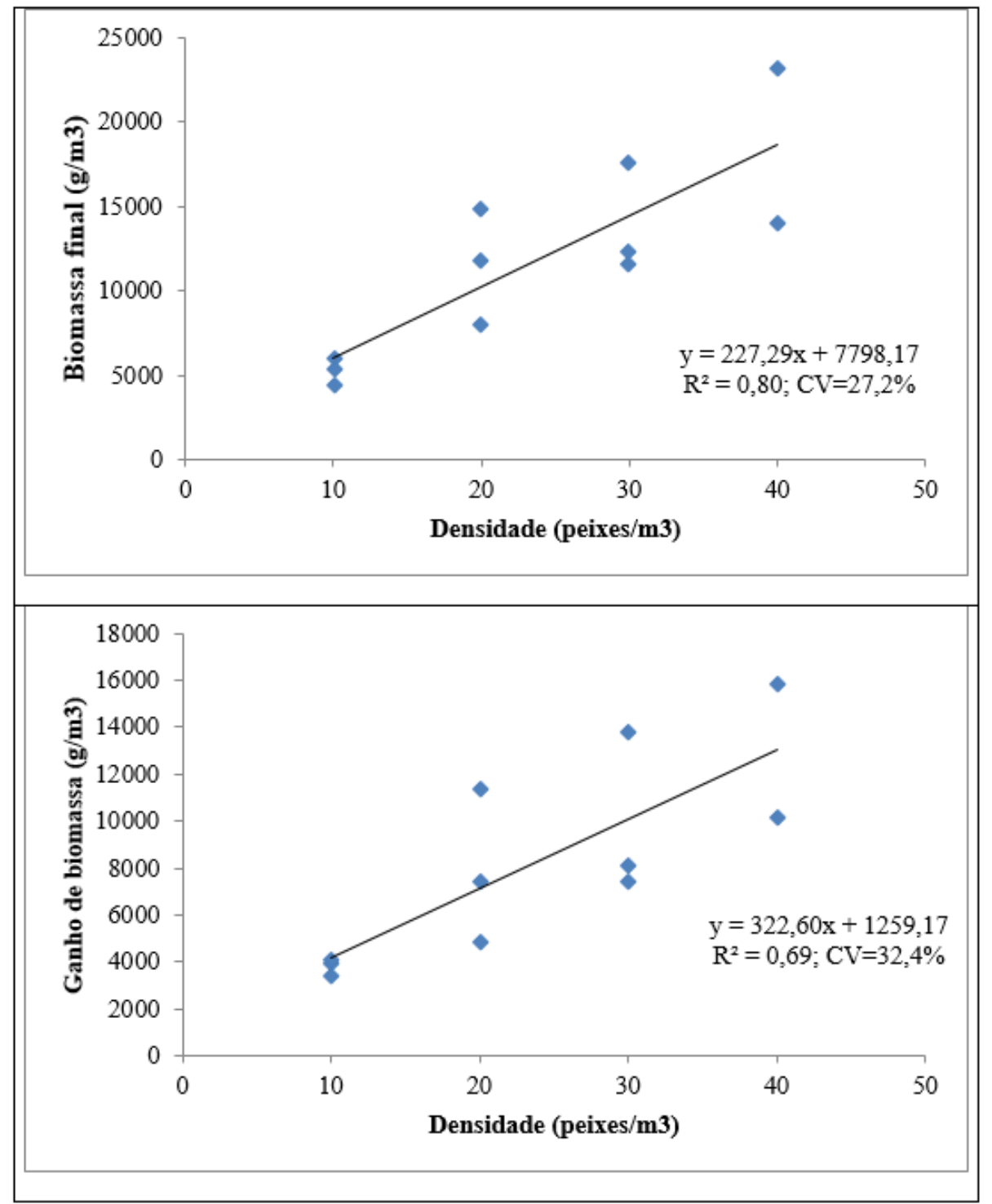

Figura 2. Efeito da densidade de estocagem do tambaqui em gaiolas de baixo volume $\left(1 \mathrm{~m}^{3}\right)$ durante 120 dias.

No presente trabalho a densidade de 40 peixes $/ \mathrm{m}^{3}$ proporcionou maior biomassa final, ganho de biomassa e ganho diário de biomassa sem alterações no crescimento, conversão alimentar aparente e demais índices zootécnicos avaliados. Chagas et al. $^{2}$ obtiveram valores semelhantes aos observados neste trabalho para ganho de peso e conversão alimentar aparente em tambaqui estocado a 15 peixes $/ \mathrm{m}^{3}$ em gaiolas de $6 \mathrm{~m}^{3}$.

$\mathrm{O}$ aumento da densidade de estocagem do tambaqui até 40 peixes $/ \mathrm{m}^{3}$ permitiu aumento do ganho de biomassa, sem alterações estatisticamente significativas da conversão alimentar aparente. Nesse contexto, o aumento da densidade de estocagem pode ser feito pelo produtor rural, dependendo dos retornos em relação ao custo da ração, o preço de venda dos peixes e as exigências dos órgãos ambientais, quanto à carga máxima de nutrientes permitida a ser liberada pelo cultivo em determinado corpo de água ${ }^{4,10}$.

No que concerne ao manejo alimentar e nutrição do tambaqui, entretanto, rações mais eficientes para o cultivo da espécie estritamente em gaiolas devem ser desenvolvidas. Os valores de conversão alimentar observados $(1,8$ a $3,5: 1)$ foram superiores aos observados em cultivos comerciais de tambaqui em viveiros escavados, que geralmente estão em torno de 1,6 a 2,2:1 ${ }^{11}$. 
O tratamento de 40 peixes $/ \mathrm{m}^{3}$ apresentou os maiores valores de biomassa, $18,6 \mathrm{~kg} / \mathrm{m}^{3}$, em 120 dias de cultivo, sem diferenças estatísticas para peso e comprimento médio final, ganho de peso e sobrevivência. A ausência de diferenças significativas entre todos os tratamentos para peso e comprimento foi provavelmente devido à grande variação de tamanho observada entre os indivíduos, que pode ser reflexo de hierarquia acentuada entre os peixes, o que pode prejudicar o acesso à comida $\mathrm{e}$ descanso aos animais mais fracos do cardume. Por isso que em cultivos comerciais em gaiolas geralmente são feitas classificações periódicas por tamanho e separação dos peixes para melhorar as condições gerais de uso das instalações e alimentação artificial, proporcionando crescimento mais adequado e menor impacto ambiental ${ }^{12}$.

Gomes et al. ${ }^{1}$ observaram a possibilidade de aumento da densidade de estocagem do tambaqui em gaiolas de $6 \mathrm{~m}^{3}$ até 50 peixes $/ \mathrm{m}^{3}$ com produção de aproximadamente $46 \mathrm{~kg} / \mathrm{m}^{3}$ em oito meses, sem alterações no crescimento dos animais, porém também com heterogeneidade de tamanho entre os peixes. Como consequência dessa hierarquia pode haver maior susceptibilidade de alguns peixes a injúrias por ataques oriundos dos peixes dominantes, que podem ainda ser portas para a maior carga de organismos ectoparasitos ${ }^{6}$. No presente estudo, no entanto, foi observado que todos os peixes analisados tinham parasitos nas brânquias sendo que $o$ tratamento de 30 peixes $/ \mathrm{m}^{3}$ apresentou os números mais elevados, mas sem diferenças significativas. Onaka et al. ${ }^{13}$ observaram níveis parecidos de parasitas nas brânquias de peixes redondos estocados em aquários, em comparação ao que foi observado no presente trabalho, com exceção para a densidade de 30 peixes $/ \mathrm{m}^{3}$.

É divulgado na literatura que o aumento da densidade de estocagem favorece a disseminação de parasitas em piscicultura ${ }^{6}$, mas isso somente foi verdadeiro até a densidade de 30 peixes $/ \mathrm{m}^{3}$ e as variações do número de parasitas nas brânquias do tambaqui foram grandes. Além disso, a incidência de monogenóides não comprometeu o crescimento, ganho de peso e sobrevivência dos animais em todos os tratamentos. No entanto, qualquer alteração na qualidade da água ou outro fator estressor podem comprometer o sistema imune destes animais, favorecendo uma maior proliferação dos parasitas ou até mesmo de lesões ocasionadas por eles que podem servir de porta de entrada para fungos e bactérias oportunistas, comprometendo dessa forma o desenvolvimento destes animais ou até sua sobrevivência ${ }^{7}$.

\section{CONCLUSÃO}

O cultivo do tambaqui em gaiolas de baixo volume apresentou melhor resultado na densidade de 40 peixes $/ \mathrm{m}^{3}$, com maior ganho de biomassa em quatro meses de cultivo. Densidade de estocagem superior proporcionou valores mais elevados de biomassa final, mas novos estudos são necessários com densidades de estocagem ainda maiores que 40 peixes $/ \mathrm{m}^{3}$ em gaiolas de baixo volume $\left(1 \mathrm{~m}^{3}\right)$ por tempo maior que 120 dias. Novos estudos também deverão ser conduzidos para o aperfeiçoamento das técnicas de cultivo do tambaqui em gaiolas de baixo volume, principalmente em respeito ao manejo da sanidade, qualidade genética, crescimento e classificação por tamanhos.

\section{AGRADECIMENTOS}

CNPQ (Processo 471655/10-4), FINEP (Projeto DARPA/SEPA/SEPROR), FAPEAM (PAIC) e EMBRAPA (Projetos MP 03.12.03.014.00.02.002; 02.12.01.020.00.00; 02.13.09.002.00.00).

\section{REFERÊNCIAS}

1. Gomes, L., Chagas, E., Martins, H., Roubach, R., Ono, E., Lourenço, J.N. Cage culture of tambaqui (Colossoma macropoтит) in a central Amazon floodplain lake. Aquaculture [Internet]. 2006 Mar [cited 2014 Sep 22]; 253(1-4): 374-384. Available from: http://www.sciencedirect.com/science/article/pii/S004484 $\underline{8605005442}$

2. Chagas, E., Gomes, L.C., Martins, H., Roubach, R. Produtividade de tambaqui criado em tanques-rede com diferentes taxas de alimentação [Tambaqui productivity reared in cages with different feeding rations]. Ciência Rural [Internet]. 2007 Jul [cited 2014 Sep 22]; 37(4): 109-1115. Available from: http://www.scielo.br/pdf/cr/v37n4/a31v37n4.pdf

3. Inoue, L.A.K.A., Boijink, C.L., Ribeiro, P.T., Silva, A.M.D., Affonso, E.G. Avaliação de respostas metabólicas do tambaqui exposto ao eugenol em banhos anestésicos [Evaluation of tambaqui metabolic responses to eugenol in anesthetics baths]. Acta Amazonica [Internet]. 2011 Jul [cited 2014 Sep 22]; 41(2): 327-332. Available

from:

http://www.scielo.br/pdf/aa/v41n2/v41n2a20

4. Kubitza, F. Os caminhos para uma piscicultura sustentável. Panorama da Aquicultura. 2010 May [cited 2014 Sep 22]; 20(119):16-23.

5. Maciel, E., Feitosa, K.C., Corrêa-Neto, C.R., Macedo, F., Mattioli, W., Abimorad, E., Abreu, J. Desempenho produtivo e parâmetros fisiológicos de juvenis de pacu criados em tanques-rede em diferentes densidades de estocagem [Performance and physiological parameters of juvenile pacu reared in cages at different stocking 
densities]. Revista Brasileira de Saúde e Produção Animal [Internet]. 2013 Jan/Mar [cited 2014 Sep 22]; 4(1):185194. Available from: http://www.scielo.br/scielo.php?pid=S1519$99402013000100022 \&$ script $=$ sci arttext

6. Baldwin, L. The effects of stocking density on fish welfare. The Plymouth Student Scientist [Internet]. 2010 Jan [cited 2014 Sep 22]; 4(1): 372-383. Available from: https://studentjournals.plymouth.ac.uk/index.php/pss/articl e/viewFile/160/203

8. Suresh, A.V., Lin, K.C. Effects of stocking density on water quality on a production of red tilapia in a recirculated water system. Aquaculture Engineering [Internet]. 1992 jan [cited 2014 Sep 22]; 11(1):1-22. Available from http://www.sciencedirect.com/science/article/pii/0144860 99290017R

9. Inoue, L.A.K.A., Senhorini, J.A., Zaniboni, E. Growth of pacu juveniles in nightly aerated systems. Acta Scientiarum Animal Sciences [Internet]. 2003 jan [cited 2014 Sep 22]; 25(1):45-48. Available from: http://www.lapad.ufsc.br/PDF/pdf0045.pdf

10. Cole, B., Boyd, C. Feeding rate, water quality and channel catfish production in ponds. Progressive Fish Culturist [Internet]. 1986 jan [cited 2014 Sep 22];48 (1): 25-29. Available from: $\underline{8640 \% 281986 \% 2948 \% 3 \mathrm{C} 25 \% 3 \mathrm{AFRWQAC} \% 3 \mathrm{E} 2.0 . \mathrm{CO}}$ \%3B2\#preview

11. Kubitza, F. Tambaqui, alimentando com eficiência para reduzir custos. Panorama da Aquicultura [Internet]. $2012 \mathrm{jan} / \mathrm{feb}$ [cited 2014 Sep 22]; 22 (129):14-21. Available from: http://www.acquaimagem.com.br/docs/Pan129_Kub tamb aqui_alimentando_eficiencia.pdf

12. Jobling, M. Simple indices for the assessment of the influences of the social environment growth performance, exemplified by studies on Artic charr. Aquaculture International [Internet]. 1995 jan [cited 2014 Sep 22]; 3(1): 60-65. Available from: http://download.springer.com/static/pdf/619/art\%253A10. 1007\%252FBF00240922.pdf?auth66=1411585828 536aa $14 \mathrm{a} 5 \mathrm{caa} 8624 \mathrm{c} 9 \mathrm{~b} 16 \mathrm{~d} 5 \mathrm{eeaa} 64 \mathrm{e} 78 \& \mathrm{ext}=. \mathrm{pdf}$

13. Onaka, E., Martins, M.L., Moraes, F. Eficácia do Albendazol e Praziquantel no controle de Anacanthorus penilabiatus (Monogenea: Dactylogiridae), parasito de pacu Piaractus mesopotamicus (Osteichthyes: Characidae) i. banhos terapêuticos [Albendazole and praziquantel efficacy against Anacanthorus penilabiatus (Monogenea: Dactylogyridae), gill parasite of Piaractus mesopotamicus (Osteichthyes: Characidae) I. Therapeutic baths]. Boletim do Instituto de Pesca [Internet]. 2003 [cited 2014 Sep 22]; 29(2):101-107. Available from: ftp://ftp.sp.gov.br/ftppesca/Onaka.pdf 